\title{
Early outcomes of stereoelectroencephalography followed by MR-guided laser interstitial thermal therapy: a paradigm for minimally invasive epilepsy surgery
}

\author{
*Kelsey Cobourn, BS, Islam Fayed, MD, Robert F. Keating, MD, and Chima O. Oluigbo, MD \\ Department of Neurosurgery, Children's National Medical Center, Washington, DC
}

OBJECTIVE Stereoelectroencephalography (sEEG) and MR-guided laser interstitial thermal therapy (MRgLITT) have both emerged as minimally invasive alternatives to open surgery for the localization and treatment of medically refractory lesional epilepsy. Although some data are available about the use of these procedures individually, reports are almost nonexistent on their use in conjunction. The authors' aim was to report early outcomes regarding efficacy and safety of SEEG followed by MRgLITT for localization and ablation of seizure foci in the pediatric population with medically refractory lesional epilepsy.

METHODS A single-center retrospective review of pediatric patients who underwent SEEG followed by MRgLITT procedures was performed. Demographic, intraoperative, and outcome data were compiled and analyzed.

RESULTS Four pediatric patients with 9 total lesions underwent sEEG followed by MRgLITT procedures between January and September 2017. The mean age at surgery was 10.75 (range 2-21) years. Two patients had tuberous sclerosis and 2 had focal cortical dysplasia. Methods of stereotaxy consisted of BrainLab VarioGuide and ROSA robotic guidance, with successful localization of seizure foci in all cases. The sEEG procedure length averaged 153 (range 67-235) minutes, with a mean of 6 (range 4-8) electrodes and 56 (range 18-84) contacts per patient. The MRgLITT procedure length averaged 223 (range 179-252) minutes. The mean duration of monitoring was 6 (range 4-8) days, and the mean total hospital stay was 8 (range 5-11) days. Over a mean follow-up duration of 9.3 (range 5.1-16) months, 3 patients were seizure free (Engel class I, 75\%), and 1 patient saw significant improvement in seizure frequency (Engel class II, $25 \%)$. There were no complications.

CONCLUSIONS These early data demonstrate that SEEG followed by MRgLITT can be used safely and effectively to localize and ablate epileptogenic foci in a minimally invasive paradigm for treatment of medically refractory lesional epilepsy in pediatric populations. Continued collection of data with extended follow-up is needed.

https://thejns.org/doi/abs/10.3171/2018.6.FOCUS18209

KEYWORDS MR-guided laser interstitial thermal therapy; MRgLITT; stereoelectroencephalography; sEEG; epilepsy; pediatric lesional epilepsy; minimally invasive epilepsy surgery; MIES

$\mathrm{E}$ PILEPSY affects 1 in 100 children, one-third of whom are unable to achieve seizure control with antiepileptic medications. ${ }^{21}$ Children diagnosed with epilepsy are at significantly increased risk for depression, anxiety, attention deficit hyperactivity disorder, conduct problems, developmental delay, autism, and headaches compared to their peers. ${ }^{33}$ Delayed seizure control is associated with progression of these impairments and a negative effect on brain development and quality of life. ${ }^{21,25,33} \mathrm{In}$ spite of these consequences, less than $1 \%$ of patients with pharmacoresistant epilepsy are referred to epilepsy centers. Of those who are referred, surgical treatment is of-

ABBREVIATIONS ECOG = electrocorticography; FCD = focal cortical dysplasia; $L O S=$ length of stay; MEG = magnetoencephalography; MIES = minimally invasive epilepsy surgery; MRE = medically refractory epilepsy; MRgLITT = MR-guided laser interstitial thermal therapy; sEEG = stereoelectroencephalography; SPGR = spoiled gradient-recalled acquisition; TDE = thermal damage estimate; TSC = tuberous sclerosis; $v E E G=$ video EEG.

SUBMITTED April 24, 2018. ACCEPTED June 15, 2018.

INCLUDE WHEN CITING DOI: 10.3171/2018.6.FOCUS18209.

${ }^{*}$ K.C. and I.F. contributed equally to this work. 
ten underutilized or significantly delayed. ${ }^{11}$ As minimally invasive techniques for the localization and treatment of foci improve, a growing number of clinicians and families have favored a surgical approach to pediatric drug-resistant focal epilepsy. ${ }^{13,27}$

Surgical treatment of epilepsy requires that the surgeon is able to 1) accurately identify the epileptogenic focus/ foci, 2) determine its relationship to eloquent structures, and 3) access the tissue for resection or lesioning. When noninvasive scalp video EEG (vEEG) monitoring is unable to identify the epileptogenic zone, more invasive localization techniques are used. Subdural strips and grids cover a wide area of the cortex, but have limited efficacy for deeper lesions. ${ }^{11}$ Similarly, open resections may be limited in their access to deep structures and in their ability to address multifocal or widespread epileptogenic networks. These challenges have led to more widespread adoption of minimally invasive techniques, including stereoelectroencephalography (sEEG) and MR-guided laser interstitial thermal therapy (MRgLITT). Early evidence supports the safety and efficacy of these procedures. ${ }^{2,6,7,9,16,18,30,31}$ However, there are currently no data describing outcomes of cases in which the two techniques have been used in concert. We hoped to demonstrate clinical scenarios that call for this method and explore its potential benefits and pitfalls.

\section{Methods \\ Study Design}

A single-center retrospective review was performed to identify all pediatric patients who underwent sEEG followed by MRgLITT between January and September 2017. All patients were treated at Children's National Medical Center in Washington, DC, by the senior author (C.O.O.). Demographic, intraoperative, and outcome data were collected and analyzed.

\section{Patient Selection}

All patients were referred to neurosurgery at Children's National Medical Center after meeting the requirements for medically refractory epilepsy (MRE), defined by the International League Against Epilepsy (ILAE) as "the failure of adequate trials of two appropriately chosen and used anti-epileptic drug schedules to achieve sustained seizure freedom." ${ }^{20}$ Each patient underwent vEEG and MRI of the brain using our 3T high-resolution epilepsy imaging protocol.

When indicated, patients underwent other ancillary studies, including functional MRI, magnetoencephalography (MEG), and FDG-PET. Cases were discussed at a multidisciplinary epilepsy conference to determine optimal management. The use of sEEG was considered for patients in whom vEEG was not sufficiently localizing, patients with suspected deep or multiple foci, and patients with no involvement of eloquent cortex requiring additional brain mapping. Laser ablation was preferred for smaller, discrete epileptogenic foci measuring less than $2-3 \mathrm{~cm}$ in diameter, especially if the foci were deep-seated and multiple. We prefer not to use laser ablation for targets that are immediately adjacent to eloquent cortex.

\section{Operative Technique for SEEG}

Prior to sEEG electrode placement, volumetric CT and MRI scans were obtained for use in trajectory planning. All procedures were performed after induction of general anesthesia with preoperative cefazolin and dexamethasone. Either the BrainLAB VarioGuide (BrainLab) or the ROSA neurosurgical robot (Medtech) were used for stereotaxy. Once trajectories were planned, stab incisions were made at the entry points. A 2.4-mm drill was used to make a twist-drill burr hole, taking into account the thickness of the bone as determined by CT. Bone anchors were placed at each entry point and depth electrodes were introduced. The wires were connected to the EEG machine and intraoperative electrocorticography $(\mathrm{ECoG})$ was used to confirm sufficient recordings at all contacts. The wires were then secured to the surrounding skin and dressings were applied. A postoperative volumetric head CT scan was obtained showing the location of the electrodes. This was merged with the preoperative volumetric MR image by using the ROSA proprietary image fusion software. This fused image allowed for the assessment of target error. Postoperative CT scan also allowed for the evaluation of possible intracranial hemorrhage. In each case, we were able to confirm that there was accurate placement of the sEEG depth electrodes as evidenced by the location of contact 1 (the deepest electrode contact) at the intended target. The patients were then admitted for monitoring until sufficient localizing data were collected. Patients were then returned to the operating room for removal of electrodes and bone anchors. In one case, the patient underwent immediate ablation after sEEG monitoring devices and electrodes were removed during the ablation procedure.

\section{Laser Ablation Procedure}

All MRgLITT procedures were performed using the Visualase system (Medtronic). In all but one case, Visualase ablation was performed 2-4 months after sEEG electrode placement. All procedures were performed after general anesthesia was induced with preoperative administration of cefazolin and dexamethasone. Stereotactic systems used included BrainLAB Neuronavigation, ClearPoint Guidance System (MRI Interventions), and the ROSA neurosurgical robot. After planning a safe trajectory from the intended entry point to the target on the applicable stereotactic planning software, 1.6-mm laser cooling tubes with metallic stylets were then carefully inserted to the measured distances and affixed to the skull using bone anchors. Once this was done, the metallic stylet was removed and the laser wire was inserted. This procedure was repeated until all the laser wires on the ipsilateral side were in position. The patient was then moved to the $1.5 \mathrm{~T}$ intraoperative MRI scanner. An initial MRI scan (T1weighted spoiled gradient-recalled acquisition [SPGR] and T2-weighted MRI) was obtained to confirm accurate placement of the laser probes. Laser ablation was then performed with real-time MRI thermography. Diffusion imaging was performed to rule out ischemia. Gadolinium was then administered for a postablation T1-weighted MRI to assure adequate ablation of the targets. The laser probes and bone anchors were then removed, the incisions 

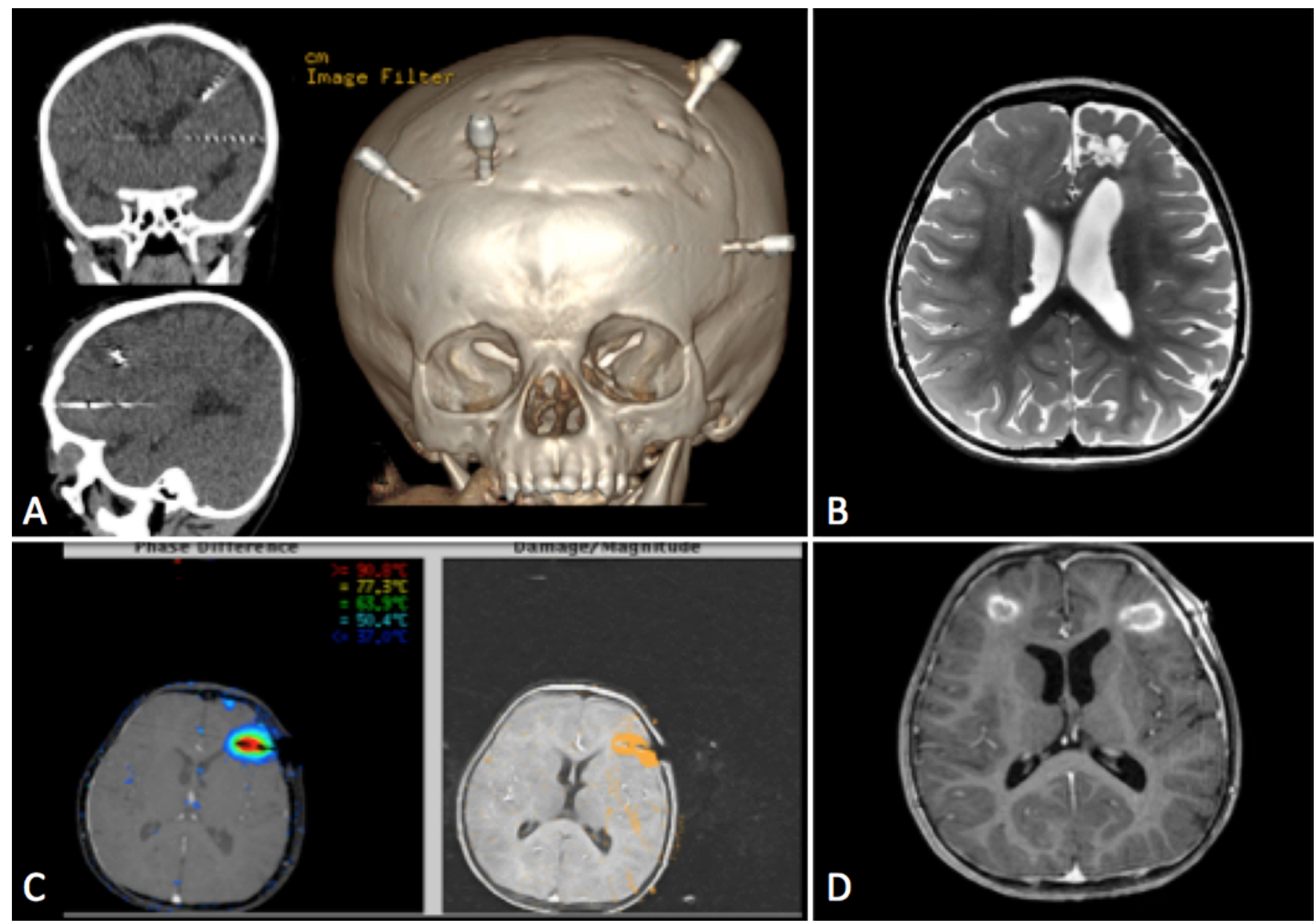

FIG. 1. Case 1. A: Head CT scan with 3D reconstruction showing sEEG depth electrodes in position. B: Preoperative T2-weighted axial brain MR image showing location of cortical tubers. C: Intraprocedure MR thermography and TDE images. D: Postablation T1-weighted SPGR axial brain MRI scan with Gd enhancement showing area of ablation.

were closed, and a final MRI sequence was obtained to assess for hemorrhage. The patient was extubated and taken to the ICU for overnight monitoring. Patients were usually discharged home within 24 hours.

\section{Case Reports}

\section{Case 1}

A 2-year-old girl with a history of tuberous sclerosis (TSC) and MRE presented to our institution. vEEG suggested a left frontal focus, which was concordant with a large left frontal tuber on MRI. After discussion of the case in a multidisciplinary epilepsy meeting, the consensus was to pursue resection with intraoperative ECoG. Postoperative MRI confirmed total resection, but the patient continued to have 15-30 seizures per day. It was thought that the focus might be a smaller left cortical tuber. Again under ECoG guidance, this smaller tuber was ablated using Visualase.

The patient's seizures persisted and MEG was performed, but could only confirm that the highest activity was in the frontal lobes. Given these data, we elected to monitor all tubers in both the left and right frontal lobes. Trajectories were planned in the ROSA workstation, using preoperative volumetric CT and MRI. Because there were 4 tubers in the frontal lobes, 4 sEEG leads were implanted.

Due to concerns about the thickness of the patient's skull and the safety of placing her in a head clamp, the leads were implanted using frameless BrainLab VarioGuide neuronavigation to avoid pinning. To confirm proper lead placement and assess for target error, postoperative CT scans showing the locations of the leads (Fig. 1A) were merged with preoperative MRI (Fig. 1B) sequences by using the proprietary ROSA image fusion software. Over 4 days of monitoring, several seizures occurred and 2 epileptogenic foci were identified-1 right superior frontal cortical tuber and 1 left inferior cortical tuber.

Given the patient's age, we elected to perform lead removal and Visualase ablation in a single procedure to avoid an additional hospital admission. This necessitated removal of the sEEG bone anchors and placement of the largercaliber Visualase bone anchors. BrainLab was again used for guidance. Following confirmation of laser wire placement on T1-weighted pre-Gd MRI, the tubers were ablated under guidance of MRI thermography and software-generated lesion maps (Fig. 1C). Following ablation, diffusion imaging ruled out ischemia and T1-weighted post-Gd MRI was obtained to confirm ablation and to detect signs of 


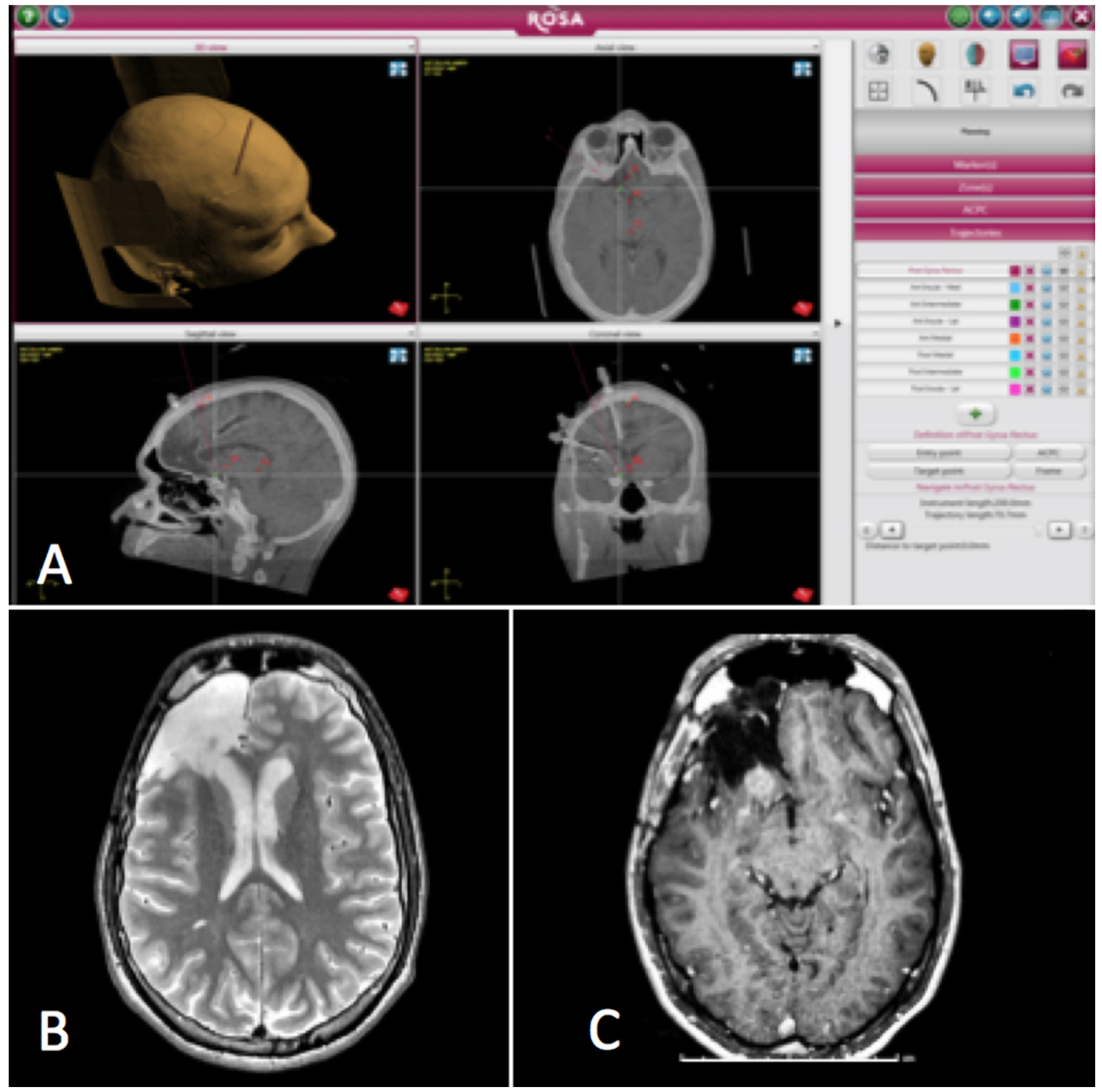

FIG. 2. Case 2. A: Screenshot from ROSA robot planning software showing planned trajectory for placement of laser probe. B: Preoperative T2-weighted axial brain MR image showing location of lesion for ablation. C: Postablation T1-weighted SPGR brain MR image with Gd enhancement showing ablated lesion in right posterior gyrus rectus.

hemorrhage (Fig. 1D). Postoperatively, the patient had no complications. She was discharged home the next day and has been seizure-free to 16 months of follow-up.

\section{Case 2}

A 21-year-old man with MRE secondary to right orbitofrontal focal cortical dysplasia (FCD) initially presented to our institution at 18 years of age, 6 years after a right partial frontal lobectomy. vEEG localized seizure origin to the right frontal and anterior temporal channels. MRI showed suspected dysplastic tissue at the posterolataeral and superomedial aspects of the original resection site. Given his unchanged seizure semiology the residual tissue was resected, with no improvement. The location of the FCD in the medial orbitofrontal cortex, very posterior on the gyrus rectus, necessitated depth electrodes to confirm the epileptogenic focus.

Following trajectory planning in the ROSA workstation (Fig. 2A), the robot was used to place 8 leads in the 

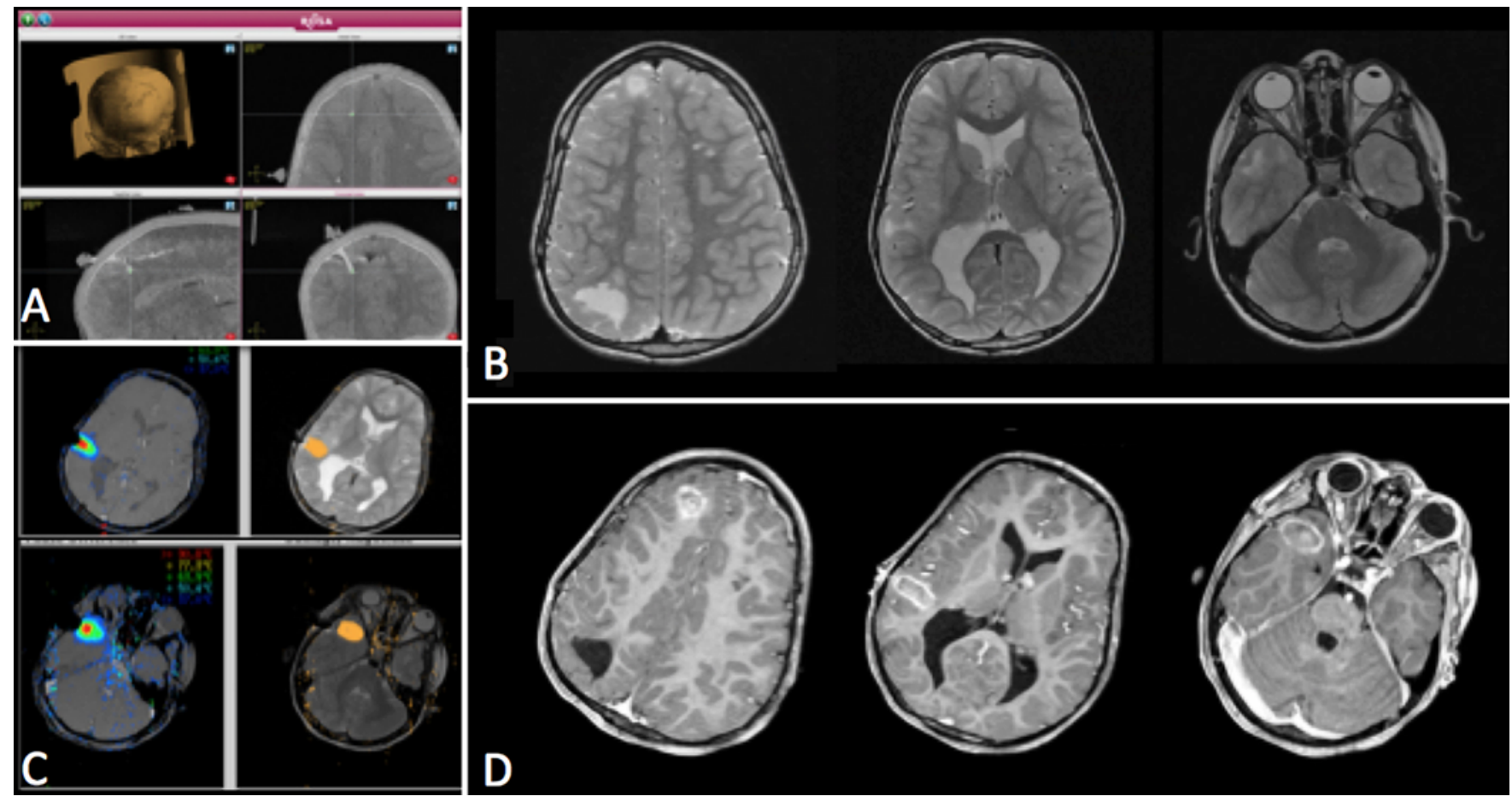

FIG. 3. Case 3. A: Screenshot from ROSA robot planning software showing planned trajectory for placement of laser probe. B: Preoperative T2-weighted axial brain MR images showing locations of lesions for ablation. C: Intraprocedure MR thermography and TDE images. D: Postablation T1-weighted SPGR brain MRIs with Gd enhancement showing the 3 ablated lesions.

right frontal lobe and right insula. Appropriate placement of depth electrodes was confirmed on postoperative brain CT imaging fused with the preoperative brain MRI scan. The preoperative T2-weighted MRI is shown in Fig. 2B. Over the 6-day monitoring period, the patient had multiple seizures arising from the remnant of the right posterior gyrus rectus, with spread to the anterior and posterior insular lateral leads.

Target selection and ablation trajectory planning were based on the locations of the sEEG electrodes. Ablation based on thermal damage mapping was performed and confirmed with T1-weighted Gd-enhanced MRI (Fig. 2C). Diffusion imaging was obtained to rule out ischemia. A final MRI sequence ruled out bleeding. The patient was discharged the following day and has remained seizure free to follow-up of 10 months.

\section{Case 3}

A 6-year-old boy with TSC presented to our center for consideration of secondary epilepsy surgery 4 years after resection of a right occipital tuber. vEEG suggested a right hemispheric onset, possibly right frontotemporal. MRI had shown numerous tubers in the right frontal and temporal lobes (Fig. 3B). We elected to use sEEG to confirm which tubers were responsible for his seizures, followed by ablation or excision.

Following computer-guided trajectory planning (Fig. $3 A)$, the ROSA robot was used to place sEEG leads. Depth electrodes were placed in tubers located in the right temporal, right frontal, and right insular lobes. Over 6 days of monitoring, 4 electroclinical seizures were seen originat- ing from the posterior temporal and the medial temporal electrode.

Ablation was performed under T2-weighted MR guidance and confirmed with Gd-enhanced T1-weighted MRI. The patient has been seizure free to follow-up of 5 months.

\section{Case 4}

A 13-year-old girl with a history of MRE and 2 previous resections of right frontal FCD was seen for consideration of invasive intracranial monitoring. Prior to treatment, the patient was having more than 100 seizures per week. With aggressive medical management, she improved to 5-8 seizures per week.

MRI showed possible FCD in the right frontal region. vEEG corroborated right frontal seizure focus. The FCD was resected with ECoG, with no improvement. Subdural strips and grids were used to localize and resect the remaining FCD. The patient was seizure-free for 1 year after surgery. She returned for consideration of additional surgery 7 years later due to increasing seizure frequency. MRI raised suspicion for residual FCD (Fig. 4B).

Her case was discussed at a multidisciplinary epilepsy meeting, with consensus that any further surgery should involve intracranial invasive monitoring via SEEG to localize the seizure focus. Four depth electrodes were placed in the right hemisphere around the prior resection cavity. Over an 8-day monitoring period, she had 5 electroclinical seizures arising from the right lateral orbitofrontal electrodes and the posterior gyrus rectus.

Ablation trajectories were planned in the ROSA workstation using preoperative scans (Fig. 4A). T1-weighted 

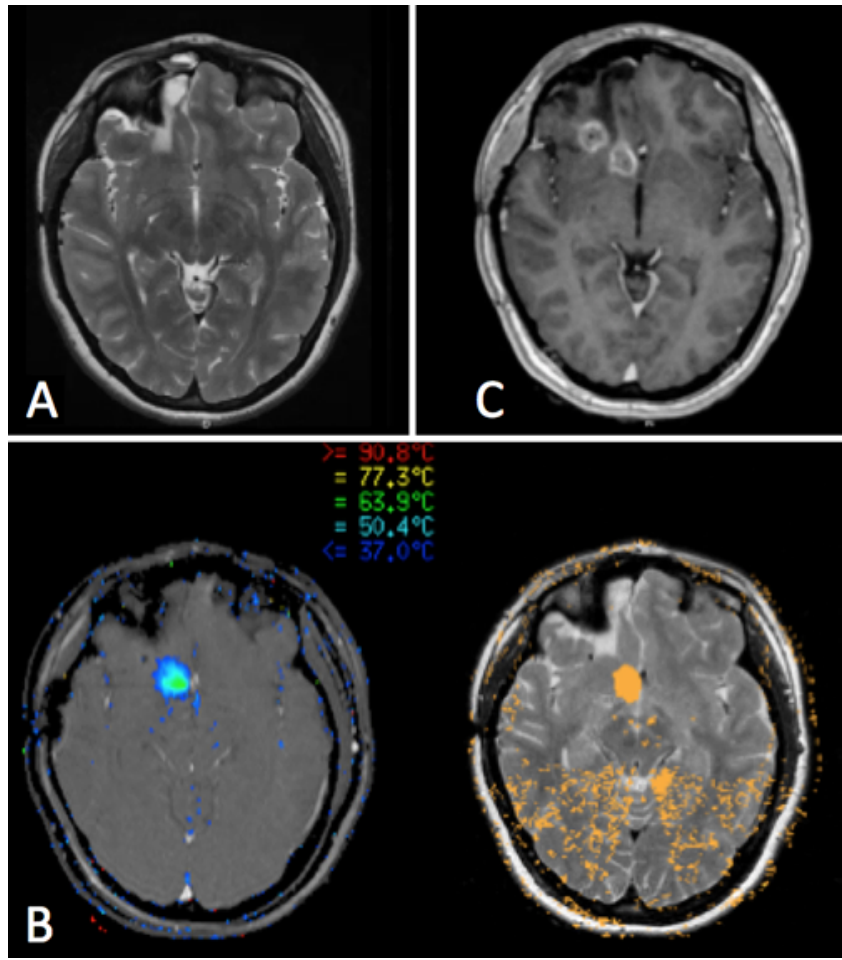

FIG. 4. Case 4. A: Preoperative T2-weighted axial brain MR image showing location of lesions for ablation. B: Intraprocedure MR thermography and TDE images. C: Postablation T1-weighted SPGR brain MR image with $\mathrm{Gd}$ enhancement showing ablated lesions.

MRI confirmed accurate placement of the wires with no signs of bleeding. The seizure foci were ablated based on thermal mapping (Fig. 4B). Having done this, T1-weighted Gd-enhanced MRI was obtained (Fig. 4C).

The patient was discharged home the next day. At 6 months of follow-up, she was no longer having her "absence" preoperative seizures, but was having generalized tonic-clonic seizures monthly. MRI showed the ablated lesions in the right posterior rectus gyrus and right lateral orbital frontal cortex. Given that the predominant preoperative seizure type had stopped, it was decided that further adjustments to her medications should be made before considering more surgery.

\section{Results}

\section{Demographic Data}

Four pediatric patients with 9 total lesions underwent sEEG followed by MRgLITT procedures between Janu- ary and September 2017. The mean age at surgery was 10.75 (range 2-21) years. Two patients had TSC and 2 had FCD. Patients were maintained on a median of 4 (range 2-6) antiepileptic drugs at the time of surgery. Patients had a median of 0 prior procedures for invasive intracranial monitoring (range $0-2$ ) and 1 prior resection (range $0-3$ ). One patient had a previous laser ablation of a tuber. Demographic data are presented in Table 1.

\section{Perioperative Data}

Methods of stereotaxy consisted of BrainLab VarioGuide and ROSA robotic guidance, with successful localization of seizure foci in all cases. The length of the sEEG procedure averaged 153 (range 67-235) minutes, with a mean of 6 (range 4-8) electrodes and 56 (range 18-84) contacts per patient. The mean duration of monitoring was 6 (range 4-8) days. The MRgLITT procedure was guided by BrainLab, ClearPoint, or ROSA. Procedure length averaged 223 (range 179-252) minutes. The mean hospital length of stay (LOS) for the ablation procedure was 2 (range 1-5) days. Perioperative data are summarized in Tables 2 and 3 .

\section{Outcome Data}

Over a mean follow-up duration of 9.3 (range 5.1-16) months, 3 patients were seizure free (Engel class I, 75\%) and 1 patient saw significant improvement in seizure frequency (Engel class II, 25\%). No ischemia, hemorrhage, focal neurological deficit, or other complications occurred in any of the patients in this series.

\section{Discussion}

Our report demonstrates the efficacy and safety of a multistage minimally invasive epilepsy surgery (MIES) paradigm leveraging a combination of sEEG and MRgLITT, as evidenced by high rates of seizure freedom with no complications in this initial cohort. Our center has performed laser ablation for epilepsy since 2013 and sEEG since our acquisition of a stereotactic neurosurgical robot in 2016. Initially, the majority of our sEEG procedures were followed by open resections. However, as our MIES practice saw increasing volume and demand, we found the next logical step to be the integration of these techniques to create a fully minimally invasive paradigm. We have found this protocol to be most useful for deep-seated foci, particularly those that do not require mapping of eloquent cortex. Although there have been several reports of sEEG and MRgLITT, evidence is sparse regarding the combination of these modalities in a multistage procedure. ${ }^{12}$ This is

TABLE 1. Characteristics for each patient in our series

\begin{tabular}{cccccccc}
\hline Case No. & Indication & Laterality & Age (yrs) & Sex & No. of AEDs & Prior Surgical Interventions & LOF (mos) \\
\hline 1 & TSC & Bilat & 2 & F & 6 & 2 & 16 \\
\hline 2 & FCD & Rt & 21 & M & 3 & 2 & 1 \\
\hline 3 & TSC & Rt & 6 & M & 2 & 3 & 5 \\
\hline 4 & FCD & Rt & 13 & F & 4 & 6 \\
\hline
\end{tabular}

AEDs = antiepileptic drugs; LOF = length of follow-up. 
TABLE 2. Perioperative data for sEEG procedure for each patient in our series

\begin{tabular}{cccccccc}
\hline Case No. & Indication & Laterality & Method of Stereotaxy & Electrodes & Contacts & Length of sEEG Procedure (mins) & LOM (days) \\
\hline 1 & TSC & Bilat & VarioGuide & 4 & 18 & 235 & 4 \\
\hline 2 & FCD & Rt & ROSA & 8 & 80 & 201 & 6 \\
\hline 3 & TSC & Rt & ROSA & 8 & 84 & 109 & 6 \\
\hline 4 & FCD & Rt & ROSA & 4 & 42 & 67 & 8 \\
\hline Avg & & & & 6 & 56 & 153 & 6 \\
\hline
\end{tabular}

Avg = average LOM = length of monitoring.

especially true in the pediatric population. This paper adds to the limited body of knowledge in this area by describing our technique and early outcomes for an interrogative and interventional epilepsy procedure performed in a completely minimally invasive fashion.

Although MIES is not a new phenomenon, it is a relatively recent player in mainstream neurosurgical practice. ${ }^{1,22,32}$ In addition to providing all patients with drug-resistant epilepsy with a wider array of options, these novel tools and techniques provide a unique opportunity for patients who may not have been surgical candidates otherwise.

sEEG has been shown to be a safe and effective procedure (Table 4). ${ }^{4-7,10,14-16,19,24,34,36,37}$ In our series, the average sEEG procedure time was 153 (range 67-235) minutes overall, and 125 (range 67-201) minutes among the cases in which robotic assistance was used. The average duration of monitoring was 6 days (range 4-8 days), with the placement of an average of 6 (range 4-8) electrodes. The average number of electrodes ranges from 5 to 18 in the literature, depending on the center., $3,5-7,10,14-16,24,34,36,37$ Given the lesional nature of our cases, the number of leads was determined by the number of lesions targeted.

Overall, the literature suggests that sEEG is a safe procedure with comparable seizure control outcomes and lower complication rates when compared with traditional invasive monitoring (Table 4). ${ }^{5-7,10,14-16,24,34,36,37}$

All patients in our series had lesional epilepsy with a median of 2.5 (range 1-3) targets. The pathological foci included tubers and FCD. The mean energy used for ablation was $2.59 \mathrm{~kJ}$ for tubers and $1.70 \mathrm{~kJ}$ for FCD. Limited reports have sought to elucidate the thermodynamics of brain tissue in response to the varied energy of ablation, ${ }^{26,28,29,35}$ but there is no normative data on the required amount of energy to ablate tissues, so surgeons must instead rely on the thermal damage estimate (TDE) on the
Visualase workstation. ${ }^{8,17,23}$ Our ablation procedures averaged 222.75 minutes (range 179-252 minutes). All patients were discharged home the day after the procedure. There were no complications during follow-up, consistent with low reported complication rates in the literature for both sEEG and MRgLITT.

In our youngest patient it was believed that the benefits of decreasing morbidity and total LOS outweighed the potential increase in infection risk. These benefits may merit offering this option to all of our patients. Although our patients did not experience any complications, it is our feeling that additional studies are needed to assess the risks and benefits. If these procedures are performed in the same admission, consideration should be given to the bore caliber of the bone anchor used for sEEG to ensure that it is sufficient to accommodate the laser cooling wire used during MRgLITT.

Although sEEG and MRgLITT are becoming well-established parts of the epilepsy surgeon's armamentarium, our report focuses on the combination of these modalities in a completely minimally invasive paradigm. From our experience, it appears that this technique is best suited for patients with multiple, discrete, widespread, lesional epileptic foci. This is particularly so when these lesions are small, first-order ellipsoid, and uncalcified. We believe the ideal case is TSC and, potentially, periventricular nodular heterotopia. ${ }^{12}$

\section{Conclusions}

The trend toward minimally invasive procedures in epilepsy surgery has provided options to patients and families seeking to improve seizure control without open surgery. It is critical to continue to adjust our approach to neurosurgical conditions that may benefit from this technology,

TABLE 3. Perioperative data for MRgLITT procedure for each patient in our series

\begin{tabular}{|c|c|c|c|c|c|c|c|}
\hline $\begin{array}{l}\text { Case } \\
\text { No. }\end{array}$ & Indication & Laterality & $\begin{array}{l}\text { Method of } \\
\text { Stereotaxy }\end{array}$ & $\begin{array}{l}\text { Lesions } \\
\text { Ablated }\end{array}$ & Energy of Ablation & $\begin{array}{l}\text { Length of Visualase } \\
\text { Procedure (mins) }\end{array}$ & $\begin{array}{l}\text { LOS } \\
\text { (days) }\end{array}$ \\
\hline 1 & TSC & Bilat & BrainLab & 2 & $10.5 \mathrm{~W} \times 165 \mathrm{~s}, 10.5 \mathrm{~W} \times 150 \mathrm{~s}$ & 179 & 5 \\
\hline 2 & FCD & Rt & ClearPoint & 1 & $10.5 \mathrm{~W} \times 178 \mathrm{~s}$ & 251 & 1 \\
\hline 3 & TSC & Rt & ROSA & 3 & $\begin{array}{l}11.25 W \times 135 s+150 s, 11.25 W \times 150 s+135 s \\
11.25 W \times 150 s+135 s\end{array}$ & 252 & 1 \\
\hline 4 & FCD & Rt & ROSA & 3 & $10.5 W \times 150 s+120 s, 10.5 W \times 135 s, 10.5 W \times 135 s$ & 209 & 1 \\
\hline Avg & & & & 2.25 & & 222.75 & 2 \\
\hline
\end{tabular}

$s=$ seconds. 
TABLE 4. Literature review suggests that sEEG is a safe and effective technique for localization of seizure focus

\begin{tabular}{|c|c|c|c|c|}
\hline Authors \& Year & No. of Patients (total/treated w/ follow-up) & Ped/Adult Patients & $\%$ Engel Class I & Complications \\
\hline Cossu et al., 2012 & $15 / 10$ & Ped & $60 \%$ & $6.7 \%$ \\
\hline Cossu et al., $2005^{6}$ & 35 & Ped & $60 \%$ & $3 \%$ \\
\hline Gonzalez-Martinez et al., $2014^{15}$ & $30 / 18$ & Ped & $55.5 \%$ & $3 \%$ \\
\hline Liava et al., 2014 & 24 & Ped & $75 \%$ & $0 \%$ \\
\hline Dylgjeri et al., 2014 & 10 & Ped & $70 \%$ & NR \\
\hline Taussig et al., 2014 & $65 / 51$ & Ped & $67 \%$ & $0 \%$ \\
\hline Cossu et al., $2005^{5}$ & $211 / 164$ & Both & $56.4 \%$ & $5.7 \%$ \\
\hline Serletis et al., 2014 & $200 / 90$ & Both & $67.8 \%$ & $2.5 \%$ \\
\hline Gonzalez-Martinez et al., $2014^{16}$ & $122 / 90$ & Both & $62 \%$ & $2.5 \%$ \\
\hline González-Martinez et al., 2016 & $100 / 68$ & Both & $66.2 \%$ & $4 \%$ \\
\hline Vadera et al., 2013 & $14 / 10$ & Both & $60 \%$ & $1 \%$ \\
\hline Avg & & & $63.6 \%$ & $2.8 \%$ \\
\hline
\end{tabular}

$\mathrm{NR}=$ not reported; ped $=$ pediatric .

especially with consideration for the unique circumstances applicable to the pediatric population. Additional experience will undoubtedly provide further insight and allow specialists to optimize these procedures and workflows.

\section{References}

1. Bancaud J: Techniques et méthodes de l'exploration fonctionnelle stéréotaxique des structures encéphaliques chez l'homme (cortex, sous-cortex, noyaux gris centraux). Rev Neurol (Paris) 101:220-227, 1959

2. Buckley R, Estronza-Ojeda S, Ojemann JG: Laser ablation in pediatric epilepsy. Neurosurg Clin N Am 27:69-78, 2016

3. Cardinale F, Casaceli G, Raneri F, Miller J, Lo Russo G: Implantation of stereoelectroencephalography electrodes: a systematic review. J Clin Neurophysiol 33:490-502, 2016

4. Cardinale F, Cossu M, Castana L, Casaceli G, Schiariti MP, Miserocchi A, et al: Stereoelectroencephalography: surgical methodology, safety, and stereotactic application accuracy in 500 procedures. Neurosurgery 72:353-366, 2013

5. Cossu M, Cardinale F, Castana L, Citterio A, Francione S, Tassi L, et al: Stereoelectroencephalography in the presurgical evaluation of focal epilepsy: a retrospective analysis of 215 procedures. Neurosurgery 57:706-718, 2005

6. Cossu M, Cardinale F, Colombo N, Mai R, Nobili L, Sartori I, et al: Stereoelectroencephalography in the presurgical evaluation of children with drug-resistant focal epilepsy. J Neurosurg 103 (4 Suppl):333-343, 2005

7. Cossu M, Schiariti M, Francione S, Fuschillo D, Gozzo F, Nobili L, et al: Stereoelectroencephalography in the presurgical evaluation of focal epilepsy in infancy and early childhood. J Neurosurg Pediatr 9:290-300, 2012

8. Curry DJ, Gowda A, McNichols RJ, Wilfong AA: MR-guided stereotactic laser ablation of epileptogenic foci in children. Epilepsy Behav 24:408-414, 2012

9. Diaz R, Ivan ME, Hanft S, Vanni S, Manzano G, Jagid J, et al: Laser interstitial thermal therapy: lighting the way to a new treatment option in neurosurgery. Neurosurgery 79 (Suppl 1):S3-S7, 2016

10. Dylgjeri S, Taussig D, Chipaux M, Lebas A, Fohlen M, Bulteau C, et al: Insular and insulo-opercular epilepsy in childhood: an SEEG study. Seizure 23:300-308, 2014

11. Engel J Jr: Why is there still doubt to cut it out? Epilepsy Curr 13:198-204, 2013
12. Esquenazi Y, Kalamangalam GP, Slater JD, Knowlton RC, Friedman E, Morris SA, et al: Stereotactic laser ablation of epileptogenic periventricular nodular heterotopia. Epilepsy Res 108:547-554, 2014

13. Freitag H, Tuxhorn I: Cognitive function in preschool children after epilepsy surgery: rationale for early intervention. Epilepsia 46:561-567, 2005

14. González-Martínez J, Bulacio J, Thompson S, Gale J, Smithason S, Najm I, et al: Technique, results, and complications related to robot-assisted stereoelectroencephalography. Neurosurgery 78:169-180, 2016

15. Gonzalez-Martinez J, Mullin J, Bulacio J, Gupta A, Enatsu R, Najm I, et al: Stereoelectroencephalography in children and adolescents with difficult-to-localize refractory focal epilepsy. Neurosurgery 75:258-268, 2014

16. Gonzalez-Martinez J, Mullin J, Vadera S, Bulacio J, Hughes G, Jones S, et al: Stereotactic placement of depth electrodes in medically intractable epilepsy. J Neurosurg 120:639-644, 2014

17. Gonzalez-Martinez J, Vadera S, Mullin J, Enatsu R, Alexopoulos AV, Patwardhan R, et al: Robot-assisted stereotactic laser ablation in medically intractable epilepsy: operative technique. Neurosurgery 10 (Suppl 2):167-173, 2014

18. Hoppe C, Witt JA, Helmstaedter C, Gasser T, Vatter H, Elger CE: Laser interstitial thermotherapy (LiTT) in epilepsy surgery. Seizure 48:45-52, 2017

19. Karsy M, Guan J, Ducis K, Bollo RJ: Emerging surgical therapies in the treatment of pediatric epilepsy. Transl Pediatr 5:67-78, 2016

20. Kwan P, Arzimanoglou A, Berg AT, Brodie MJ, Allen Hauser W, Mathern G, et al: Definition of drug resistant epilepsy: consensus proposal by the ad hoc Task Force of the ILAE Commission on Therapeutic Strategies. Epilepsia 51:10691077,2010

21. Kwan P, Schachter SC, Brodie MJ: Drug-resistant epilepsy. N Engl J Med 365:919-926, 2011

22. LaRiviere MJ, Gross RE: Stereotactic laser ablation for medically intractable epilepsy: the next generation of minimally invasive epilepsy surgery. Front Surg 3:64, 2016

23. Lewis EC, Weil AG, Duchowny M, Bhatia S, Ragheb J, Miller I: MR-guided laser interstitial thermal therapy for pediatric drug-resistant lesional epilepsy. Epilepsia 56:1590-1598, 2015

24. Liava A, Mai R, Tassi L, Cossu M, Sartori I, Nobili L, et al: Paediatric epilepsy surgery in the posterior cortex: a study of 62 cases. Epileptic Disord 16:141-164, 2014 
25. McCagh J, Fisk JE, Baker GA: Epilepsy, psychosocial and cognitive functioning. Epilepsy Res 86:1-14, 2009

26. Munier SM, Hargreaves EL, Patel NV, Danish SF: Effects of variable power on tissue ablation dynamics during magnetic resonance-guided laser-induced thermal therapy with the Visualase system. Int J Hyperthermia [epub ahead of print], 2017

27. Naegele J: Epilepsy and the plastic mind. Epilepsy Curr 9:166-169, 2009

28. Patel NV, Frenchu K, Danish SF: Does the thermal damage estimate correlate with the magnetic resonance imaging predicted ablation size after laser interstitial thermal therapy? Oper Neurosurg [epub ahead of print], 2017

29. Patel NV, Jethwa PR, Barrese JC, Hargreaves EL, Danish SF: Volumetric trends associated with MRI-guided laser-induced thermal therapy (LITT) for intracranial tumors. Lasers Surg Med 45:362-369, 2013

30. Patel P, Patel NV, Danish SF: Intracranial MR-guided laserinduced thermal therapy: single-center experience with the Visualase thermal therapy system. J Neurosurg 125:853860, 2016

31. Ravindra VM, Sweney MT, Bollo RJ: Recent developments in the surgical management of paediatric epilepsy. Arch Dis Child 102:760-766, 2017

32. Reisch R, Stadie A, Kockro RA, Hopf N: The keyhole concept in neurosurgery. World Neurosurg 79 (2 Suppl): 17. e9-17.e13, 2013

33. Russ SA, Larson K, Halfon N: A national profile of childhood epilepsy and seizure disorder. Pediatrics 129:256-264, 2012

34. Serletis D, Bulacio J, Bingaman W, Najm I, GonzálezMartínez J: The stereotactic approach for mapping epileptic networks: a prospective study of 200 patients. J Neurosurg 121:1239-1246, 2014
35. Sun XR, Patel NV, Danish SF: Tissue ablation dynamics during magnetic resonance-guided, laser-induced thermal therapy. Neurosurgery 77:51-58, 2015

36. Taussig D, Chipaux M, Lebas A, Fohlen M, Bulteau C, Ternier J, et al: Stereo-electroencephalography (SEEG) in 65 children: an effective and safe diagnostic method for pre-surgical diagnosis, independent of age. Epileptic Disord 16:280-295, 2014

37. Vadera S, Mullin J, Bulacio J, Najm I, Bingaman W, Gonzalez-Martinez J: Stereoelectroencephalography following subdural grid placement for difficult to localize epilepsy. Neurosurgery 72:723-729, 2013

\section{Disclosures}

The authors report no conflict of interest concerning the materials or methods used in this study or the findings specified in this paper.

\section{Author Contributions}

Conception and design: Oluigbo, Fayed. Acquisition of data: Fayed. Analysis and interpretation of data: Cobourn, Fayed. Drafting the article: Oluigbo, Cobourn. Critically revising the article: Oluigbo, Cobourn, Fayed. Reviewed submitted version of manuscript: Oluigbo, Cobourn, Keating. Approved the final version of the manuscript on behalf of all authors: Oluigbo. Administrative/technical/material support: Oluigbo, Keating. Study supervision: Oluigbo, Keating.

\section{Correspondence}

Chima O. Oluigbo: Children's National Medical Center, Washington, DC. coluigbo@cnmc.org. 